\title{
Optimizing Outcomes in Bariatric Surgery: A Primer in Patient Selection
}

\author{
Natan Zundel, ${ }^{1}$ Sayeed Ikramuddin, ${ }^{2}$ and Raul Rosenthal ${ }^{3}$ \\ ${ }^{1}$ Minimally Invasive and Bariatric Surgery, FSFB, Bogata, Colombia \\ ${ }^{2}$ Department of Surgery, University of Minnesota Medical School, Minneapolis, MN 55455, USA \\ ${ }^{3}$ Cleveland Clinic Florida, Weston, USA \\ Correspondence should be addressed to Natan Zundel, drnazuma99@yahoo.com
}

Received 14 October 2012; Accepted 14 October 2012

Copyright (C) 2012 Natan Zundel et al. This is an open access article distributed under the Creative Commons Attribution License, which permits unrestricted use, distribution, and reproduction in any medium, provided the original work is properly cited.

We are very pleased to introduce a series of six individual papers addressing important areas in bariatric and metabolic surgery. Particularly, these papers highlight patient selection, preparation for surgery, and optimizing outcomes. The metabolic benefits of surgery are well established, but adequate patient selection can help realize better outcomes for our patient population. The first paper reviews the impact of inflammatory gene polymorphisms on bioelectrical parameters in obese subjects specifically looks at outcomes of singlenucleotide polymorphisms of IL- 6 on body composition following the laparoscopic adjustable gastric banding. The authors have demonstrated that these polymorphisms lead to improved outcomes following bariatric surgery whereas gene-wide association and screening is not an accepted modality today; it certainly will be something that is easily and readily available for patients in the next few years and will be obtainable in a pharmaceutical store.

Y. F. Wei and colleagues evaluate the candidacy of morbidly obese patients with pulmonary dysfunction for bariatric surgery. Pulmonary dysfunction is a broad topic and classically it has been understood that the impact on sleep apnea is a dynamic and rapid improvement in many patients, but not all patients; however, other diseases such as pulmonary fibrosis pose significant clinical problems for a number of surgeons and in this context the authors provide some meaningful insight into care in this patient population.

D. K. Kadeli and colleagues address an important question that asks payers, policy makers, and surgeons alike, what is the role of preoperative weight loss and its impact on long-term weight loss at one year? Whereas the authors acknowledge that a low-calorie diet preoperatively can enhance perioperative safety by reducing the size of the liver and physiologically stabilizing the patient, this specific review addresses the notion of preoperative weight loss impacting long-term weight loss. The results are intriguing in that they suggest that initial weight, that is, weight at the time of surgery, indicates an important starting point with a weight loss proportional to that at the time of nadir suggesting that weight loss preoperatively may, in fact, result in improved outcomes postoperatively at one year.

Fourth, D. A. Becker and colleagues examined the neurologic complications of nutrition deficiency following bariatric surgery. Whereas the incidence of this appears to be low, nutritional deficiencies can result in significant potential neurologic compromise in these patients. The authors outlined the best evidence in terms of outcome and some of the more esoteric yet clinically relevant aspects of neurologic deficiency and how these can be addressed, diagnosed, and managed.

C. E. Owers and colleagues address steps to optimize perioperative care of patients undergoing bariatric surgery. Here, the authors discussed the important role of lowcalorie diets in shrinking the liver, the importance of the use of a multidisciplinary team in evaluation of patients, and appropriate control of comorbid illnesses. This is an excellent review of salient topics in this area.

Finally, an important area that has not been addressed adequately in the literature to date is the impact of the sleeve gastrectomy on a super morbid obese group. J. M. Catheline and colleagues review their outcomes on weight in this population demonstrating durable and significant outcomes of the sleeve gastrectomy in the super obese population. 
In this group of 30 patients, in which they had followup on 23 at 18 months, $77 \%$ of patients were defined to have sufficient weight loss, with insufficient weight loss in the remaining six patients requiring either resleeve or conversion to a gastric bypass. This suggests that for super obesity a more robust operation may be indicated as a first procedure and that certainly these patients are amenable to an operation such as the duodenal switch.

\section{Natan Zundel Sayeed Ikramuddin \\ Raul Rosenthal}




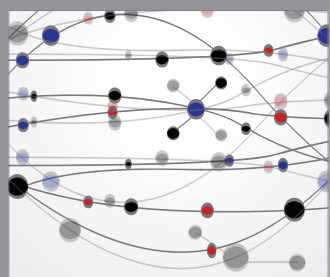

The Scientific World Journal
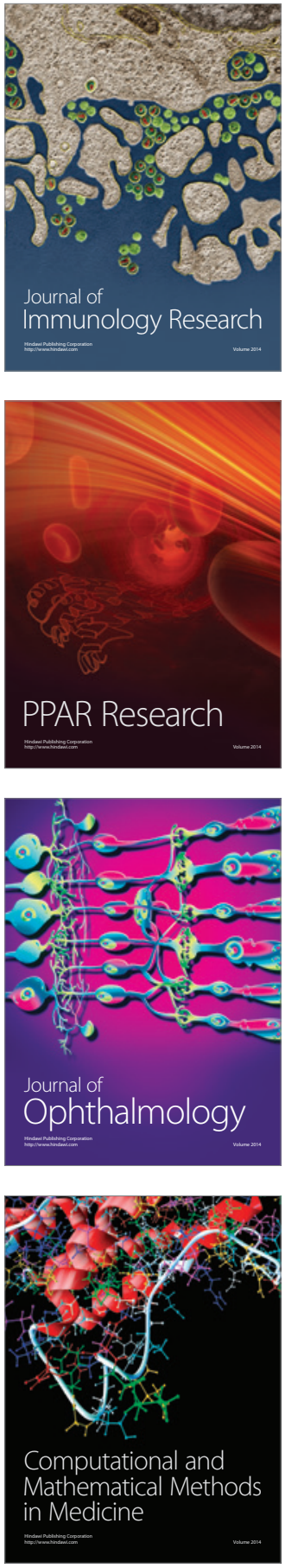

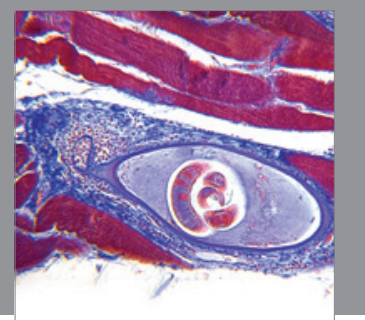

Gastroenterology

Research and Practice
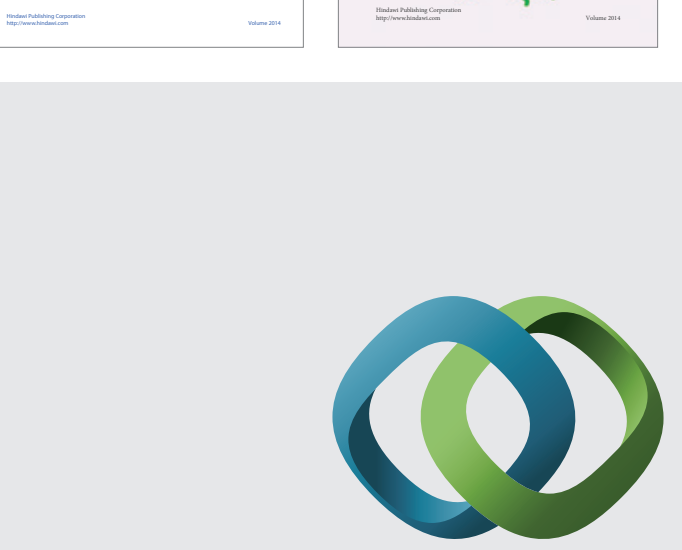

\section{Hindawi}

Submit your manuscripts at

http://www.hindawi.com
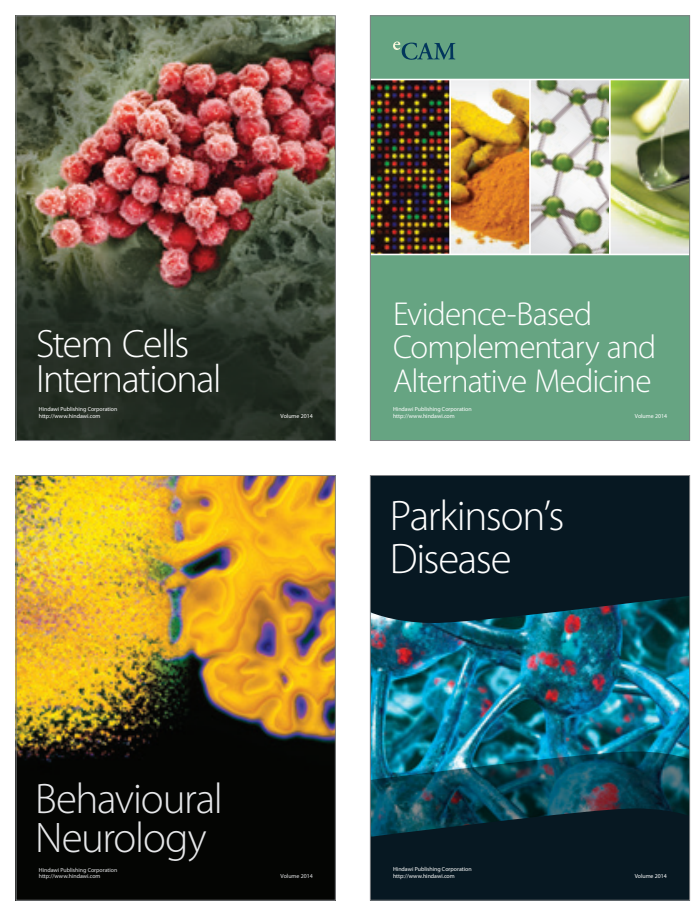

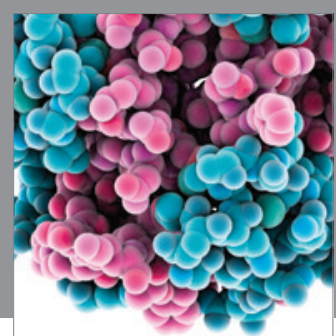

Journal of
Diabetes Research

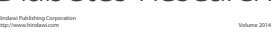

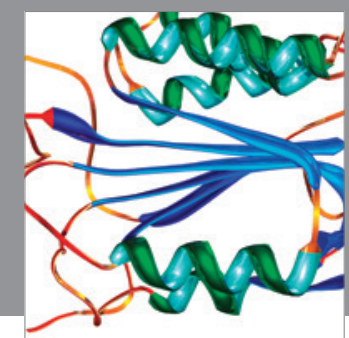

Disease Markers
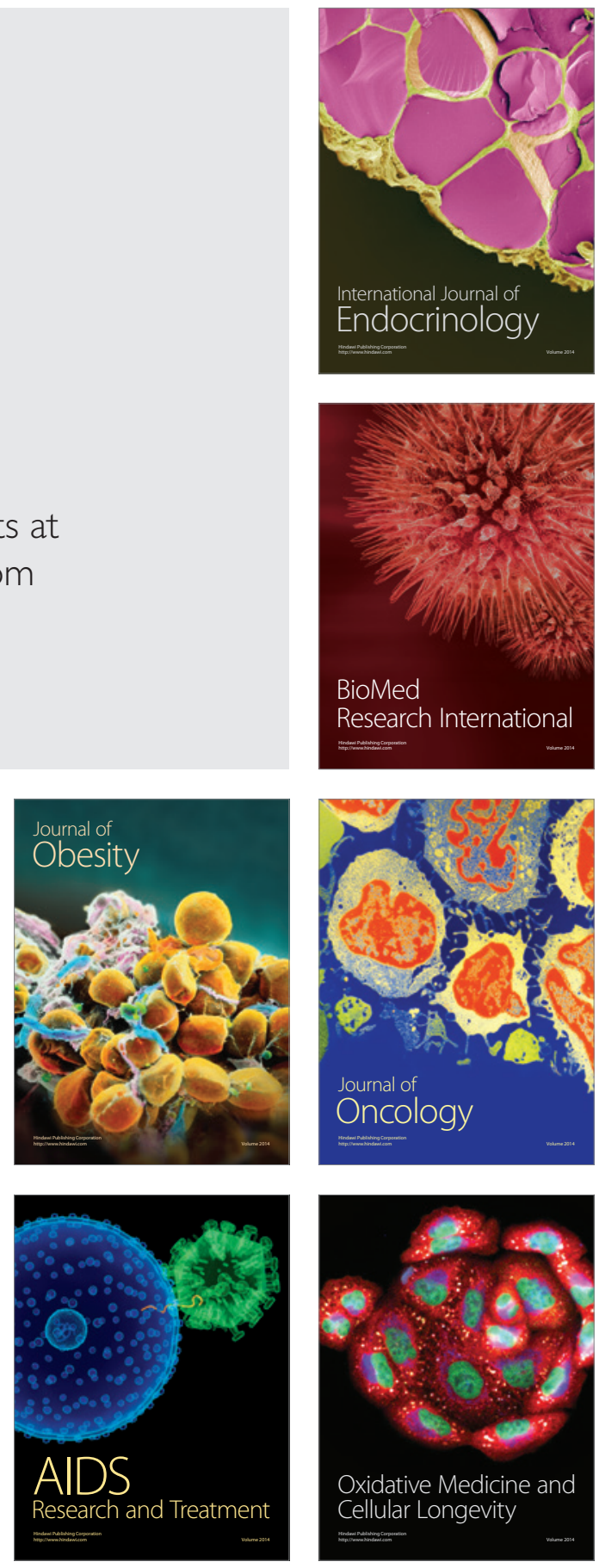